\title{
BMJ Open A systematic review protocol for examining 30-day readmission costs for atrial fibrillation patients
}

\author{
Taylor-Jade Woods (D) , ${ }^{1}$ Peter Speck, ${ }^{2}$ Billingsley Kaambwa ${ }^{1}$
}

To cite: Woods T-J, Speck P, Kaambwa B. A systematic review protocol for examining 30-day readmission costs for atrial fibrillation patients. BMJ Open 2019;9:e032101. doi:10.1136/ bmjopen-2019-032101

- Prepublication history and additional material for this paper are available online. To view these files, please visit the journal online (http://dx.doi. org/10.1136/bmjopen-2019032101).

Received 03 June 2019 Revised 20 September 2019 Accepted 23 September 2019

Check for updates

(C) Author(s) (or their employer(s)) 2019. Re-use permitted under CC BY-NC. No commercial re-use. See rights and permissions. Published by BMJ.

${ }^{1}$ College of Medicine and Public Health, Flinders University, Adelaide, South Australia, Australia

${ }^{2}$ College of Science and Engineering, Flinders University, Adelaide, South Australia, Australia

Correspondence to Taylor-Jade Woods; wood0302@flinders.edu.au

\begin{abstract}
Introduction Atrial fibrillation (AF) is the most common sustained cardiac arrhythmia and readmissions of AF patients place a huge burden on the healthcare system, including economically. With an increasing prevalence, the burden of AF will continue evolving. To illuminate the readmission-specific economic burden, we aim to provide quality evidence on the cost of readmissions within 30 days where $\mathrm{AF}$ has been the primary diagnosis at the index admission.
\end{abstract}

Methods and analysis We will conduct a systematic review of all peer-reviewed articles examining readmission costs for AF patients. We will search MedLine, Cumulative Index to Nursing and Allied Health Literature, Scopus and Cochrane Library for articles written in English, published in peer-reviewed journals from inception to 2019. Reporting of this protocol follows the Preferred Reporting Items for Systematic Reviews and MetaAnalyses Protocols checklist. Studies will be included if patients were aged 18 years and over, AF was the primary diagnosis of index admission and costs of readmission within 30 days were reported. Quality assessment of studies will be done using a modified Evers checklist. Study results will be summarised in a Forest plot and heterogeneity tested for using the Cochran's $Q$ and ${ }^{2}$ statistic. A random-effects model will be applied for metaanalysis if studies are sufficiently homogeneous. The cost of readmission to hospital within 30 days for AF patients is the main outcome of interest while additional outcomes are 30-day readmission rate, predictors of readmission and predictors of readmission costs.

Ethics and dissemination Formal ethical approval is not required as no patients will be involved. Dissemination of results will be through a peer-reviewed publication. PROSPERO registration number CRD42019132017

\section{INTRODUCTION}

Atrial fibrillation (AF) is a cardiac arrhythmia ${ }^{1}$ sustained by irregular electrical activity in the heart. ${ }^{2}$ Asynchronous atrial contraction and ventricular excitation affect the ejection of blood into circulation. ${ }^{3}$ Without pharmacological or surgical intervention, or direct-current cardioversion to terminate the dysrhythmia, AF progresses and episodes of electrical disturbance become regular and eventually permanent. ${ }^{2}$ In cases of permanent $\mathrm{AF}$, restoration of sinus rhythm is either

\section{Strengths and limitations of this study}

Systematic review protocol of published peer-reviewed articles following Preferred Reporting Items for Systematic Reviews and Meta-Analyses Protocols guidelines.

- Provides highest level of evidence to decision makers.

- Informs on the economic impact of readmission in atrial fibrillation (AF) patients.

- Availability of high-quality studies examining the costs and readmission rates of AF patients may be limited.

- Varying cost inclusions and methods of cost comparisons limit opportunity for subgroup analyses.

difficult or inadvisable. ${ }^{2}$ The progressive evolution of $\mathrm{AF}$ is underscored by electrical, structural and anatomical remodelling of the atria which causes functional impairment of the heart. ${ }^{24}$ Electrical remodelling alters ion channel expression and/or function and contributes to drug resistance, recurrence following cardioversion and progression to persistent $\mathrm{AF}^{2}{ }^{2}$ Structural remodelling presents as fibrotic separation of muscle fascicles and slowing of electrical conductance with replacement of dead cardiomyocytes and headways permanent $\mathrm{AF}^{2}$ Anatomic remodelling affects the discharge of ions and action potentials in autonomic nervous signalling, establishing a positive-feedback loop. ${ }^{2}$

The absolute prevalence of AF has increased over time, showing an age-related gradient in which patients aged 85 years and over are most at risk and comprise 9\%-10\% of all cases. ${ }^{4}$ Alongside a rise in prevalence, the number of hospitalisations of AF patients has increased and readmission accounts for a large proportion of these. ${ }^{5}$ Unplanned readmissions are recognised as a safety and quality indicator in healthcare, ${ }^{6}$ because they often result from failure to provide adequate care during the prior admission. ${ }^{7}$ International literature consistently reports a high number of 30-day readmissions for AF patients, ${ }^{8-10}$ 
including after surgical intervention. ${ }^{11-13}$ Iñiguez Vázquez et $a l^{8}$ compared readmission between non-AF and AF patients in Lugo, Spain and found AF patients were readmitted more frequently than non-AF patients. A retrospective cohort study in the USA found that $22.7 \%$ of newly diagnosed $\mathrm{AF}$ and $17.6 \%$ of chronic AF patients were readmitted in 30 days postdischarge, ${ }^{10}$ suggesting that $\mathrm{AF}$ patients have been poorly managed during the index admission. Hence, identifying predictors of readmission becomes important to identify patients are high risk of readmission.

To understand resource use dedicated to managing $\mathrm{AF}$ patients during readmission, it is important to examine the economic impact on the healthcare system. Coyne et al examined three federally funded USA databases for costs of hospital treatment of non-valvular AF. ${ }^{14}$ The results showed the overall national cost of treatment was US $\$ 6.65$ billion, and $44 \%$ (US $\$ 2.93$ billion) was specifically dedicated to hospitalisations where AF was the primary diagnosis. ${ }^{14}$ Based on prospective data collection from the Euro Heart Survey enrolling 5333 patients, inpatient care and interventional procedures consumed $>70 \%$ of total annual costs and were main drivers of increase costs. ${ }^{15}$ At a patient level, annual direct costs were estimated at approximately US $\$ 10100$ to US\$14200 in the USA and $€ 450$ to $€ 3000$ in Western Europe per patient per year. ${ }^{16}$ Therefore, the economic burden of AF-related readmissions is significant at the national and patient level, and when taken into consideration alongside the number of readmissions, AF patients are clearly in need of improved management.

Readmissions have become a growing focus of decision and policy makers with various funding models developed around the world to provide incentive to reduce readmission. In the UK, a non-payment policy introduced in 2011 ensures readmission above hospital-specific readmission rates are not funded. ${ }^{6}$ The Hospital Readmission Risk Reduction Program for US Medicare patients penalises hospitals at a fixed percentage if their readmission rate is higher than the risk-adjusted benchmark. ${ }^{6}$ In Australia, a series of three funding options designed to financially penalise readmissions will be trialled for 2 years from July 2019 while providing incentive to improve care during the index or prior admission. ${ }^{6}$

The evolving burden AF places on healthcare systems currently requires significant hospital and economic resources and the rising prevalence of $\mathrm{AF}$ indicates this will continue. Readmissions in particular consume a large portion of healthcare resources while being central to policy reform. Accordingly, this systematic review protocol will aim to examine the costs of readmissions following an index admission where $\mathrm{AF}$ has been the primary diagnosis.

\section{METHODS AND ANALYSIS}

This systematic review protocol is registered with PROSPERO and reported according to the Preferred Reporting
Items for Systematic Reviews and Meta-Analyses Protocols checklist ${ }^{17}$ presented as online supplementary file 1 . The systematic review will be conducted based on a modification of the Cochrane Handbook for Systematic Reviews of Interventions, ${ }^{18}$ given the topic of review is costs, rather than intervention. The completed review will be reported according to the Preferred Reporting Items for Systematic Reviews and Meta-Analyses (PRISMA) guidelines. ${ }^{19}$

\section{Population}

The systematic review will focus on the costs of readmissions to hospital 30 days following discharge at index admission where AF has been the primary diagnosis. Reporting of a primary diagnosis of AF at index admission should be in the section identifying the population of eligible texts. Primary diagnoses are commonly defined using the International Statistical Classification of Diseases and Related Health Problems 9th or 10th revision codes. The population will be limited to patients aged 18 years and over. If patient age has not been reported or the primary diagnosis for the index admission was not specified or could not be discerned, the study will be excluded.

\section{Study design}

Studies will be included if costs associated with hospital readmission within 30 days of discharge where $\mathrm{AF}$ was the primary diagnosis at index admission have been reported. Peer-reviewed articles will be included unless the full text cannot be obtained. Any letters, conference papers, abstracts, editorials, reviews, theory papers, theses or dissertations will be excluded.

\section{Search strategy}

The search strategy has been developed by the authors with assistance from a research services librarian. Draft search strategies for all databases are included in online supplementary file 2. A second independent librarian will review the final search strategy against the Peer Review of Electronic Search Strategy checklist. ${ }^{20}$ The search strategy aims to retrieve published cost analyses written in English (figure 1) from all countries reporting readmission costs associated with AF patients. We will search MedLine (via Ovid), Cumulative Index to Nursing and Allied Health Literature, Cochrane Library and Scopus databases with no limit on publication date to capture all cost analyses published from inception to 2019. In addition to synonymous textwords, 'readmission', 'atrial fibrillation' and 'cost' will be key Medical Subject Headings used to capture the study population and key outcomes of interest. To identify additional papers, Google Scholar and hand searches of reference lists will be used. Further, the Australia and New Zealand Clinical Trials Register and ClinicalTrials.gov websites will be searched for clinical trials examining readmission costs in parallel with published results in peer-reviewed journals.

\section{Study selection}

Citations retrieved from the search will be managed in EndNote and duplicates removed. Two independent 
1. Retrieval of literature published in peer-reviewed journals in English from MedLine (via Ovid), CINAHL, Cochrane and Scopus databases using $\mathrm{MeSH}$ and textword searches including atrial fibrillation, readmission and cost synonyms. No limits on publication date.

2. Hand search of reference lists, Google Scholar and clinical trials registers to identify additional publications.

3. Collation of articles in Endnote and duplicates removed.

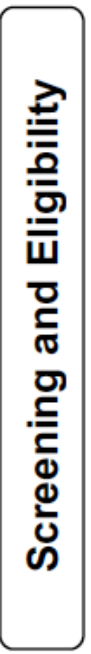

1. Peer-reviewed articles reporting readmission costs of patients aged 18 and over discharged at index admission with a primary diagnosis of AF will be included.

2. Dual review of all titles and abstracts.

3. Two reviewers will independently screen all eligible full-texts and interrater agreement will be measured using Cohen's kappa statistic.

4. Reporting of study selection using a PRISMA flow diagram.

5. Quality assessment of full-texts to be included in final analysis using modified Evers checklist.

1. Extraction of pre-specified data by one reviewer and crosschecking by a second independent reviewer.

2. Pooling of study results into a Forest plot.

3. Assessment of heterogeneity of effect measures using Cochran's $Q$ and $l^{2}$.

4. Random-effects meta-analysis if studies are sufficiently homogenous $\left(I^{2}>50 \%\right)$.

5. Narrative synthesis of data structured on the basis of data extraction protocol.

6. Cost of readmission to hospital within 30 days following discharge with primary diagnosis of $\mathrm{AF}$ the main outcome of interest. Additional outcomes include 30 -day readmission rates, predictors of readmission and predictors of readmission costs.

Figure 1 Schematic of the key systematic review processes. AF, atrial fibrillation; CINAHL, Cumulative Index to Nursing and Allied Health Literature; MeSH, Medical Subject Heading; PRISMA: Preferred Reporting Items for Systematic Reviews and Meta-Analyses.

reviewers will screen all titles and abstracts for eligibility against the inclusion/exclusion criteria. Two reviewers will independently screen all eligible full texts. Interrater agreement will be calculated using Cohen's kappa statistic. ${ }^{21}$ Agreement of $>80 \%$ (strong level of agreement) is desirable. ${ }^{21}{ }^{22}$ Selection discrepancies will be resolved through discussion and independent assessment by a third reviewer, if needed. This process will be reported using a PRISMA flow diagram.

Quality assessment

A modified Evers checklist ${ }^{23}$ will be used to assess methodological validity of full-text studies selected for inclusion. Because no standardised or validated checklist specifically 
for cost studies exists, modification of the Evers checklist ${ }^{23}$ is required to reflect that the studies retrieved are not full economic evaluations. The modified Evers checklist is presented in online supplementary file 3 .

\section{Data extraction}

Data will be extracted by one reviewer and cross-checked independently by a second reviewer. Disagreements will be resolved by discussion and consultation with a third reviewer, if required. Data items to extract will be relevant to the review question to facilitate a narrative synthesis. These items include time period of study, study setting (eg, country, primary care) or source of data (eg, database used); study design; number of eligible patients; causes of readmission captured (eg, all-cause, AF-relayed) and how they were identified (eg, International Statistical Classification of Diseases and Related Health Problems, 10th Revision code); subgroups compared (eg, all-cause vs AF-related readmission, primary vs secondary diagnosis of $\mathrm{AF}$ ); reporting of costs (eg, currency and year, conversion rates); cost components/costs included; statistical analysis; number of readmissions within 30 days; predictors of readmission identified; predictors of costs; overall cost results. Cost results will be inflated to a common currency and currency year using national or international Consumer Price Index inflation indices where appropriate.

\section{Outcomes}

The main outcome of interest is the cost of readmission to hospital within 30 days following discharge where $\mathrm{AF}$ was the primary diagnosis at index admission. This will include relevant monetary values. Cost comparisons may be different between papers, for example, measured between the index admission versus readmission or between consecutive readmissions. Additional outcomes include 30-day readmission rate, predictors of readmission and predictors of readmission costs if reported.

\section{Data analysis}

If possible, data will be pooled and reported in a Forest plot to visually summarise the results of each study. Heterogeneity of effect measures will be assessed using Cochran's $Q$ test and quantified using the $\mathrm{I}^{2}$ statistic. An $\mathrm{I}^{2}$ value of $>50 \%$ will be considered to show substantial heterogeneity as differences between study results are beyond those attributable to chance alone. ${ }^{24}$ If studies are sufficiently homogenous, we will apply a random-effects model to better deal with different effect sizes between studies. Cost data collected from specific jurisdictions is considered the best resource to assist in decision-making regarding resource allocation in the local context. ${ }^{25}$ Therefore, grouping studies based on income level, for example, will maintain comparability. However, we anticipate meta-analysis may not be possible due to a small number of eligible studies and the sensitivity of cost data to specific settings which limits transferability of cost estimates. ${ }^{26}$
Formal subgroup analysis will depend on the available data in articles included in the final analysis and cannot be specified in advance. To evaluate the studies included in the final analysis, a narrative synthesis of the data will be undertaken. This will be structured around the data extraction protocol with a focus on readmission costs.

\section{Patient and public involvement}

No patients were involved in the design of this protocol. No patients will participate in recruitment or conduct of this study as it is a protocol for a systematic review. For this reason, dissemination of the results to participants is not applicable.

\section{CONCLUSION}

This review will provide evidence on the costs of 30-day readmissions of AF, the most common cardiac arrhythmia. The economic impact of resource use dedicated to these readmissions will be drawn from direct cost measurements. Other outcomes including readmission rates will illuminate whether resource use delivers meaningful benefits to patients and improves cardiovascular care outcomes. Identifying predictors of readmissions and readmission costs will be used to understand the types of patients requiring better outcome management. Overall, this review will complement existing evidence on AF readmissions and their impact.

Acknowledgements The authors would like to acknowledge Research Services librarian Mrs Shannon Brown for her assistance in conceiving the search strategy.

Contributors T-JW, PS and BK conceived the idea and design of the protocol. T-JW designed the figure and wrote the first draft and is the guarantor of the manuscript. All authors read, provided feedback and approved the final written manuscript.

Funding T-JW is a recipient of an Honours scholarship from the Playford Memorial Trust Inc. and Fay Fuller Foundation. The funding source had no involvement in study design; in the collection, analysis and interpretation of data; in the writing of the report; and in the decision to submit the protocol for publication.

Competing interests None declared.

Patient consent for publication Not required.

Provenance and peer review Not commissioned; externally peer reviewed.

Data availability statement All data relevant to the study are included in the article or uploaded as supplementary information.

Open access This is an open access article distributed in accordance with the Creative Commons Attribution Non Commercial (CC BY-NC 4.0) license, which permits others to distribute, remix, adapt, build upon this work non-commercially, and license their derivative works on different terms, provided the original work is properly cited, appropriate credit is given, any changes made indicated, and the use is non-commercial. See: http://creativecommons.org/licenses/by-nc/4.0/.

ORCID iD

Taylor-Jade Woods http://orcid.org/0000-0002-0545-0401

\section{REFERENCES}

1 Brieger D, Amerena J, Attia J, et al. National heart Foundation of Australia and the cardiac Society of Australia and New Zealand: Australian clinical guidelines for the diagnosis and management of atrial fibrillation 2018. Heart, Lung and Circulation 2018;27:1209-66.

2 Iwasaki Y-ki, Nishida K, Kato T, et al. Atrial fibrillation pathophysiology: implications for management. Circulation 2011;124:2264-74. 
3 Staerk L, Sherer JA, Ko D, et al. Atrial fibrillation: epidemiology, pathophysiology, and clinical outcomes. Circ Res 2017;120:1501-17.

4 Ball J, Carrington MJ, McMurray JJV, et al. Atrial fibrillation: profile and burden of an evolving epidemic in the 21st century. Int $\mathrm{J}$ Cardiol 2013;167:1807-24.

5 Briffa T, Hung J, Knuiman M, et al. Trends in incidence and prevalence of hospitalization for atrial fibrillation and associated mortality in Western Australia, 1995-2010. Int J Cardiol 2016;208:19-25.

6 Independent Hospital Pricing Authority (IHPA). Consultation paper on the pricing framework for Australian public hospital services 2019-20. Sydney, NSW IHPA; 2018.

7 Considine J, Fox K, Plunkett D, et al. Factors associated with unplanned readmissions in a major Australian health service. Aust. Health Review 2019;43:1-9.

8 Iñiguez Vázquez I, Monte Secades R, Matesanz Fernández M, et al Characteristics and temporal pattern of readmissions of patients with atrial fibrillation hospitalized in medical departments. Rev Clin Esp 2017;217:309-14.

9 Freeman JV, Wang Y, Akar J, et al. National trends in atrial fibrillation hospitalization, readmission, and mortality for Medicare beneficiaries, 1999-2013. Circulation 2017;135:1227-39.

$10 \mathrm{Kim} \mathrm{MH}$, Lin J, Hussein M, et al. Incidence and temporal pattern of hospital readmissions for patients with atrial fibrillation. Curr Med Res Opin 2009;25:1215-20.

11 Franzini C, Chee KW, Chen L, et al. 30-Day readmission following atrial fibrillation catheter ablation. Can J Cardiol 2013;29.

12 Noseworthy PA, Kapa S, Haas LR, et al. Trends and predictors of readmission after catheter ablation for atrial fibrillation, 2009-2013. Am Heart J 2015;170:483-9.

13 Arora S, Lahewala S, Tripathi B, et al. Causes and predictors of readmission in patients with atrial fibrillation undergoing catheter ablation: a national population-based cohort study. J Am Heart Assoc 2018;7:e009294.

14 Coyne KS, Paramore C, Grandy S, et al. Assessing the direct costs of treating nonvalvular atrial fibrillation in the United States. Value Health 2006:9:348-56.
15 Ringborg A, Nieuwlaat R, Lindgren P, et al. Costs of atrial fibrillation in five European countries: results from the Euro heart survey on atrial fibrillation. Europace 2008;10:403-11.

16 Wolowacz SE, Samuel M, Brennan VK, et al. The cost of illness of atrial fibrillation: a systematic review of the recent literature. Europace 2011;13:1375-85.

17 Shamseer L, Moher D, Clarke M, et al. Preferred reporting items for systematic review and meta-analysis protocols (PRISMA-P) 2015: elaboration and explanation. BMJ 2015;349:g7647.

18 Higgins J, Green S. Cochrane Handbook for systematic reviews of interventions version 5.1.0: the Cochrane collaboration, 2011. Available: www.handbook.cochrane.org

19 Liberati A, Altman DG, Tetzlaff J, et al. The PRISMA statement for reporting systematic reviews and meta-analyses of studies that evaluate healthcare interventions: explanation and elaboration. $B M J$ 2009;339:b2700.

20 McGowan J, Sampson M, Salzwedel DM, et al. PRESS Peer Review of Electronic Search Strategies: 2015 Guideline Statement. J Clin Epidemiol 2016;75:40-6.

21 Cohen J. A coefficient of agreement for nominal scales. Educ Psychol Meas 1960;20:37-46.

22 McHugh ML. Interrater reliability: the kappa statistic. Biochem Med 2012;22:276-82.

23 Evers S, Goossens $\mathrm{M}$, de Vet $\mathrm{H}$, et al. Criteria list for assessment of methodological quality of economic evaluations: consensus on health economic criteria. Int J Technol Assess Health Care 2005;21:240-5.

24 Higgins JPT, Thompson SG, Deeks JJ, et al. Measuring inconsistency in meta-analyses. BMJ 2003;327:557-60.

25 Cooper N, Coyle D, Abrams K, et al. Use of evidence in decision models: an appraisal of health technology assessments in the UK since 1997. J Health Serv Res Policy 2005;10:245-50.

26 Sculpher M, Pang F, Manca A, et al. Generalisability in economic evaluation studies in healthcare: a review and case studies. Health Technol Assess 2004:8:1-192. 